\title{
Transdiscal mid- and upper thoracic vertebroplasty: first description of 2 exemplary cases
}

\author{
Andreas K. Filis, MD, ${ }^{1}$ Kamran Aghayev, MD, ${ }^{1}$ Bernhard Schaller, MD, PhD, DSc, ${ }^{2}$ \\ Jennifer Luksza, PA-C, and Frank D. Vrionis, MD, PhD' \\ 'Department of Neuro-Oncology, H. Lee Moffitt Cancer Center and Research Institute, Morsani College of Medicine, \\ University of South Florida, Tampa, Florida; and 2University of Oxford, United Kingdom
}

\begin{abstract}
Kyphoplasty and vertebroplasty are established treatment methods to reinforce fractured vertebral bodies. In cases of previous pedicle screw instrumentation, vertebral body cannulation may be challenging. The authors describe, for the first time, an approach through the adjacent inferior vertebra and disc space in the thoracic spine for cement augmentation. A 78-year-old woman underwent posterior fusion with pedicle screws after vertebrectomy and reconstruction with cement and Steinmann pins for a pathological T-7 fracture. Two months later she developed a compression fracture of the vertebral body at the lower part of the construct, and a vertebroplasty was performed. Because a standard transpedicular route was not available, an inferior transdiscal trajectory was used for the cement injection. A 73-year-old man with a history of rheumatoid arthritis underwent cervicothoracic fusion posteriorly for subluxation. He developed pain in the upper thoracic area, and the authors performed a transdiscal vertebroplasty at T-2. The standard transpedicular route was not possible. The vertebral body was satisfactorily filled up with cement. Clinically both patients benefited significantly in terms of back pain and showed an uneventful follow-up of 3 months. Transdiscal vertebroplasty can achieve good results in the mid- and upper thoracic spine when a standard transpedicular trajectory is not possible, and can therefore be a good alternative in select cases.
\end{abstract}

http://thejns.org/doi/abs/10.3171/2015.12.SPINE15946

KEY WORDS kyphoplasty; vertebroplasty; fusion; transdiscal trajectory; thoracic; technique

$\mathrm{V}$ ERTEBROPLASTY is an established surgical method of reinforcing vertebral bodies with compression fractures (either osteoporotic or pathological). The standard trajectory for cannulation is bilateral transpedicular. However, there are other clinical scenarios when this route is not applicable. The extrapedicular approach has been described as an alternative approach for those cases. ${ }^{10}$ Except for these procedures in the lumbar spine, the thoracic spine poses special neuroanatomical challenges, considering the relatively small pedicle size and severe angulation from physiological kyphosis in the mid- and upper thoracic spine.

The transdiscal trajectory for stabilization has been reported with the introduction of the transdiscal screw in the lumbar spine. ${ }^{1,7}$ In 2004, Mehdizade et al. reported percutaneous vertebroplasty via a transdiscal route following transpedicular instrumentation in the lumbar spine. ${ }^{6}$ For the thoracic spine, such a procedure has not yet been described. In this paper we therefore present, for the first time, 2 cases of the transdiscal technique in the mid- and upper thoracic spine and give some special attention to the potential indication of this relatively new method.

\section{Case Reports}

Case 1

A 78-year-old woman with history of multiple myeloma presented with back pain and progressive paraparesis. The MRI showed a compression fracture at T-7 with epidural disease and spinal cord compression (Fig. 1). She underwent a T-7 laminectomy, transpedicular bilateral T-7 vertebrectomy, vertebral body reconstruction with polymethylmethacrylate (PMMA) and Steinmann pins, and a T5-9 posterior transpedicular stabilization and fusion. Figure 2 shows postoperative results with correction of the kyphotic deformity and physiological alignment. The neurological condition of the patient improved, and she was discharged home.

Two months later the patient presented with increasing kyphotic deformity and wound ulceration at the lower part

ABBREVIATIONS PMMA $=$ polymethylmethacrylate.

SUBMITTED August 8, 2015. ACCEPTED December 17, 2015.

INCLUDE WHEN CITING Published online March 11, 2016; DOI: 10.3171/2015.12.SPINE15946. 

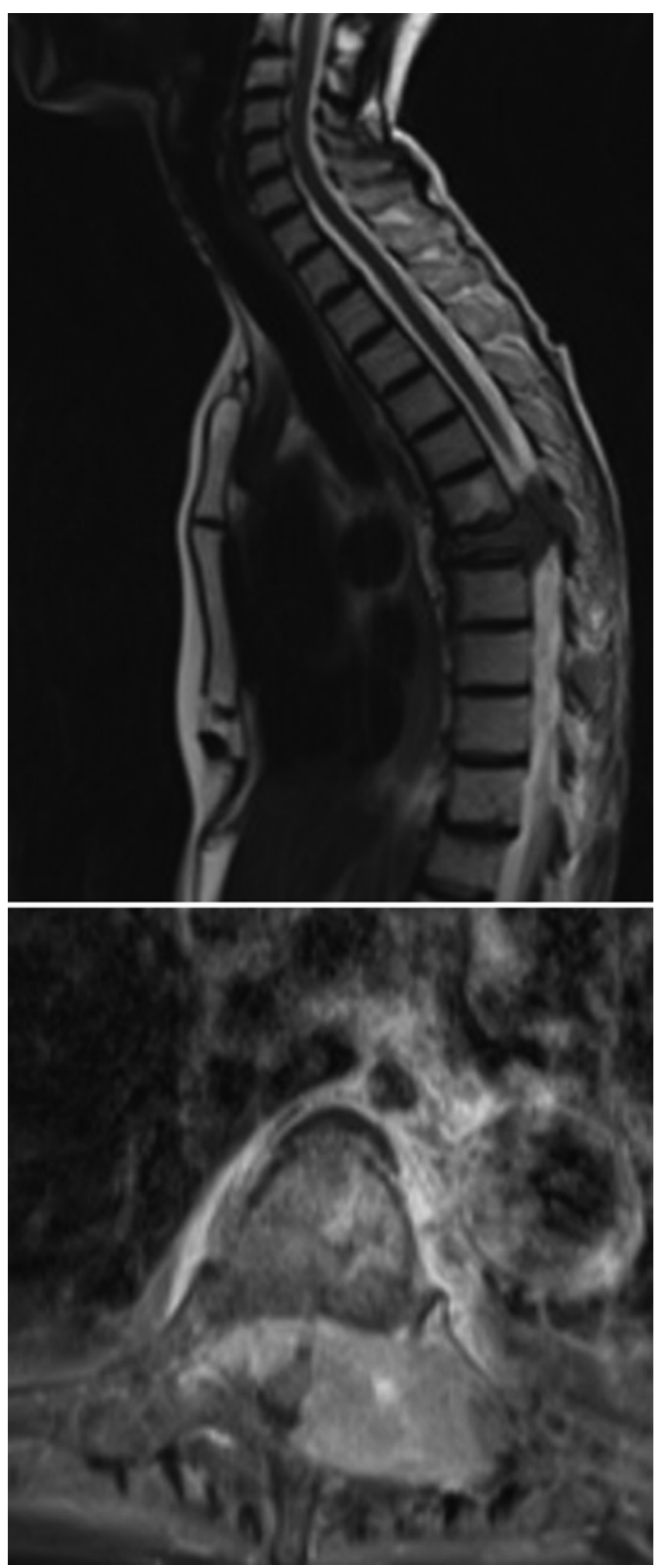

FIG. 1. Case 1. Preoperative sagittal T2-weighted (upper) and axial postcontrast T1-weighted (lower) MR images showing a T-7 compression fracture with epidural disease compressing the spinal cord. of the construct on the left side with pain and no neurological deficit. The plain lateral radiograph (Fig. 2) and MR image of the thoracic spine revealed compression fractures of the T-9 vertebra at the lower part of the construct. The segmental kyphosis resulted in dorsal displacement of the rods' inferior end points, causing a $1-\mathrm{cm}$ wound compromise. Specifically, the wound was not infected, and the hardware was not exposed. After discussion with the patient and her family the decision was made to proceed with vertebral augmentation to avoid further kyphotic deformity, followed by open surgery to remove the lower part of the hardware, and wound debridement. Due to the patient's extremely poor nutritional status, those procedures were performed in 2 stages.

The cannulation of T-10 was performed in a standard transpedicular fashion using the StabiliT system (Dfine Inc.). At T-10 we performed a unilateral approach and advanced the Jamshidi needle to the middle of the vertebral body, which was symmetrically filled with the PMMA cement. For the T-9 level, however, the option of a transpedicular route was not feasible. We proceeded from the contralateral T-10 vertebral pedicle through the T9-10 disc space into the body of the T-9 vertebra (Fig. 3). The starting point at the skin level was caudal to the T-10 pedicle, approximately $1 \mathrm{~cm}$ lateral to the lateral border of the pedicle on the anteroposterior scan. The pedicle entrance started at the inferolateroposterior aspect of the pedicle to proceed cranially, medially, and anteriorly. Specific attention was given to make sure that the tip of the Jamshidi needle was ventral to the spinal canal as it was advanced beyond the medial border of the pedicle. The needle was advanced into the disc space and then to the T-9 vertebral body. After filling the anterior and middle portions of T-9, we pulled out the Jamshidi needle. There was slight extravasation of PMMA cement into the T9-10 disc space, presumably through the defect created by the Jamshidi needle in the inferior endplate of T-9 (Fig. 3).

The patient was discharged home after the surgery. Upon follow-up she did not show any signs of progressive kyphosis. The patient later underwent the second stage of surgery as previously mentioned. The lower part of the hardware, the T-9 pedicle screws, and lower portions of the rods were removed. The wound healed without complications. Unfortunately, 3 months later the patient's disease progressed, and she chose to proceed with hospice placement.

\section{Case 2}

A 73-year-old man with a history of severe rheumatoid arthritis after a fall developed predominantly upperextremity paresthesia and pain in the cervical area (visual analog scale score of 10 without pain medication and 5 with medication, and an Oswestry Disability Index of 33). Radiographically there was evidence of swan neck deformity (Fig. 4A) with anterior subluxation most prominent at levels C6-7 and C7-T1. We proceeded with a C2-7 laminectomy and posterior fusion from $\mathrm{C}-2$ to $\mathrm{T}-2$ (Fig. 4B). Postoperatively the patient's preoperative symptoms improved significantly.

Six months later the patient developed a wound dehiscence at the upper thoracic area. Part of the cause was 

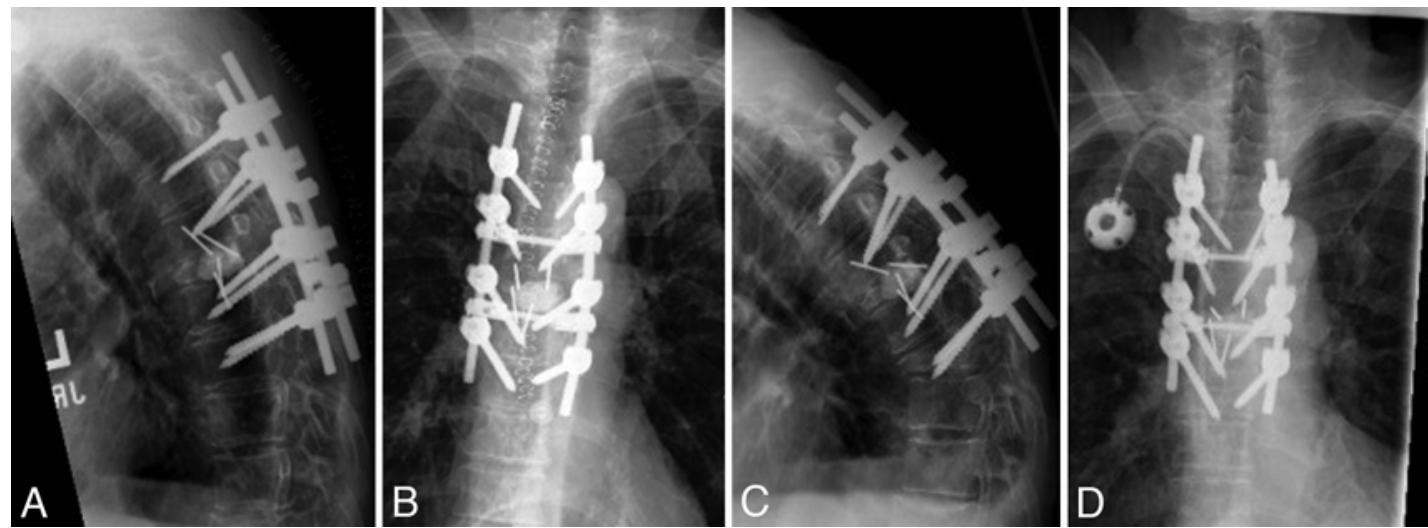

FIG. 2. Case 1. Early (A and B) and late (C and D) lateral and anteroposterior plain radiographs showing the interval development of a T-9 compression fracture with segmental kyphosis and posterior displacement of the rods' inferior end points.

muscle atrophy, which resulted in hardware being in contact with skin, leading inevitably to dehiscence. After unsuccessful long-standing wound care we performed wound debridement with a myofacial right trapezius flap. In the follow-up clinic appointments the wound was now closed and dry.
Four months after the second surgery the patient presented again in the clinic, this time with complaints of increasing pain in the upper thoracic area. Radiographically there was no hardware failure, but given the patient's poor bone quality with osteoporosis from long-standing steroid use because of the rheumatoid arthritis, we performed a
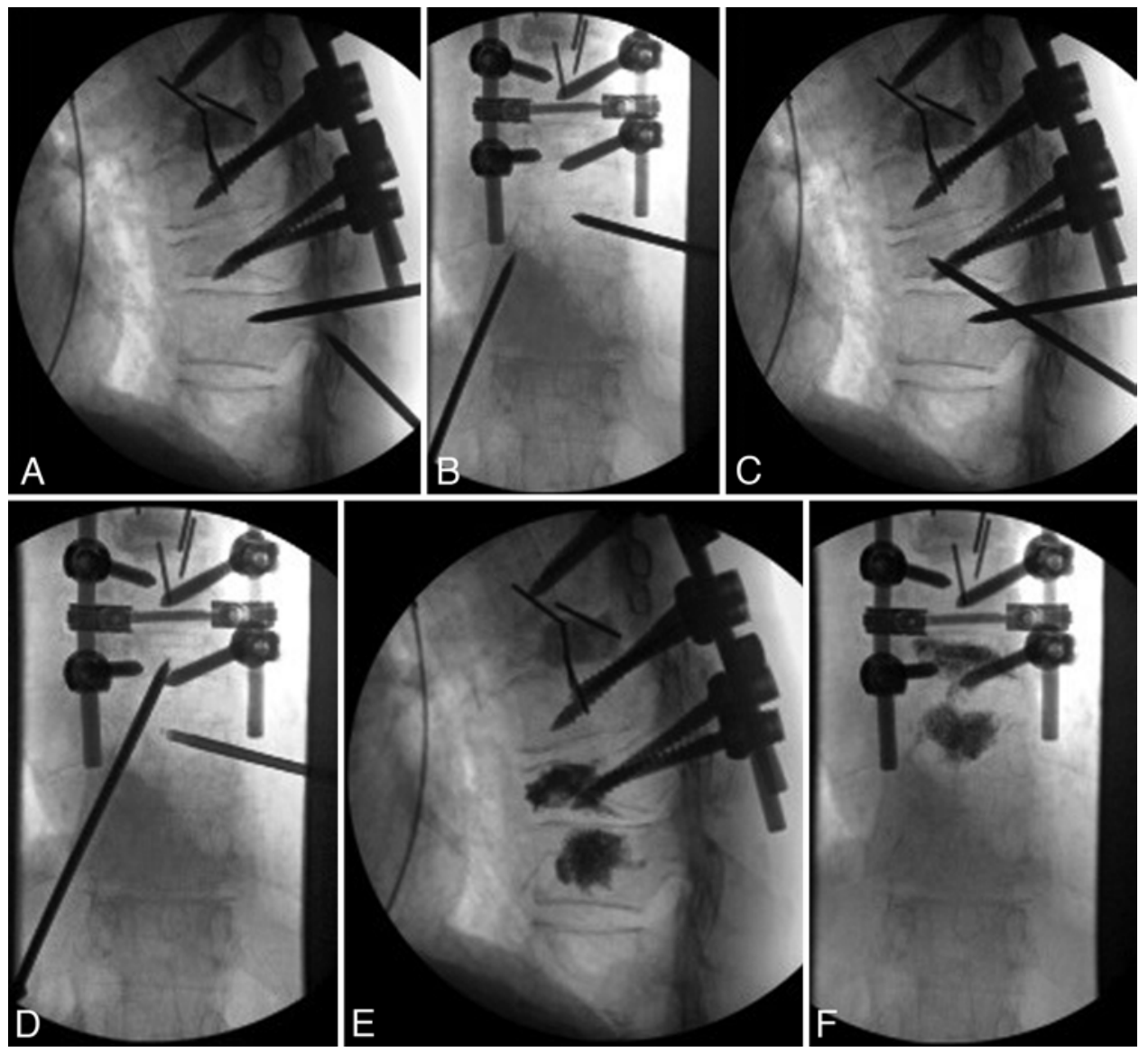

FIG. 3. Case 1. Anteroposterior and lateral fluoroscopic images during the procedure. A and B: The T-10 vertebral body has been cannulated. The bone starting point for the transdiscal cannulation is inferoposterolateral to the pedicle. C and D: A needle is placed into the mid-T-9 body. E and F: Images obtained after cement injection show adequate filling with small cement extravasation into the T9-10 disc space. 

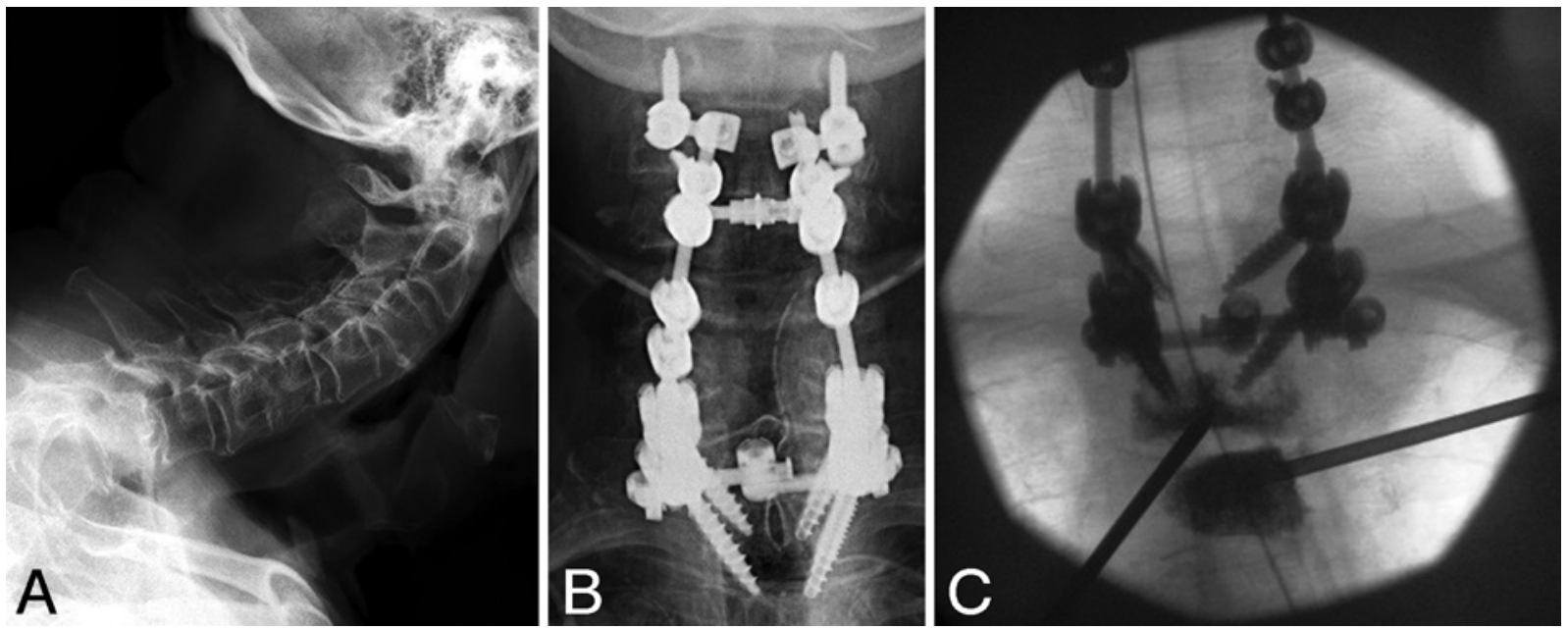

FIG. 4. Case 2. A: Preoperative lateral radiograph of the cervical and upper thoracic spine showing significant swan neck deformity with anterior subluxations. B: Postoperative anteroposterior radiograph showing posterior fusion with screws and rods from C-2 to T-2. C: Intraoperative fluoroscopic image showing transdiscal cannulation of the T-2 vertebra and transpedicular cannulation of the T-3 vertebra. Cement has been injected into the vertebral bodies.

T-2 and T-3 kyphoplasty. Because of the aforementioned wound situation we chose a lower skin incision for a T-2 transdiscal and T-3 transpedicular vertebroplasty (Fig. 4C). Postoperatively the pain improved significantly; there was no new wound dehiscence.

\section{Discussion}

Compression fracture of a previously instrumented vertebral body is not a rare clinical situation, but especially in long constructs and osteoporotic patients, the treatment is not straightforward. The standard treatment options, vertebroplasty or kyphoplasty, are technically challenging. There is always the risk of pneumothorax, hematoma secondary to injury of the segmental vessels, embolism of PMMA, or leakage.

Ge et al. and Ryu et al. have reported cases with balloon kyphoplasty using the extrapedicular approach.,11 But even if the extrapedicular approach is used, starting far laterally or using Kambin's triangle, it is difficult to manipulate the Jamshidi needle for optimal placement as the pedicle screws interfere with the needle, especially in the mid- and upper thoracic spine. Therefore, only the transdiscal cannulation starting from adjacent vertebrae, as described in the presented 2 cases, provides the necessary angle to avoid the screws in this spine location and bring the Jamshidi needle to the middle of the vertebral body. Only with this newly described technique can one perform vertebroplasty to the most cranial thoracic vertebra of the fused segment. Using such a transdiscal trajectory, it is also possible to perform balloon kyphoplasty. In our cases we used the StabiliT system, which includes an osteotome to create a cavity in the vertebral bone instead of the classic balloon dilation. Because of the existing pedicle screws we did not use the osteotome. To date, cannulation of the instrumented vertebrae in the middle of a construct has not been described in the literature.

It is advisable to avoid extravasation into the disc space, although this does not have clinical implications other than an increased risk of adjacent-level vertebral fracture. The surgeon should be watchful, most importantly on the lateral fluoroscopy, not to cause retropulsion of cement. Due to the small number of published cases in the literature it is not clear if transdiscal vertebroplasty is riskier than standard approaches with regards to cement extravasation. In our first case, PMMA leakage into the disc space occurred after we withdrew the Jamshidi needle and was probably due to the inferior T-9 endplate defect. It might be advisable to keep the Jamshidi needle in place for some time to allow the PMMA to harden in the vertebral body to prevent this type of leakage.

It should also be emphasized that puncture and exposure to cement may cause disc degeneration. It is well known that simple disc puncture, even with a small gauge needle, causes accelerated degeneration. ${ }^{5,8,12}$ Mao et al. in a rabbit model demonstrated that exposure to PMMA further speeds up disc degeneration. ${ }^{4}$ These factors should be considered before choosing the transdiscal route for vertebral augmentation. In our patient the transdiscal access was selected due to the short life expectancy of the patient and relative immobility of the thoracic segments.

Transdiscal trajectory has initially been reported for stabilization of high-grade spondylolisthesis at the L5-S1 level. ${ }^{1,7}$ The transdiscal screw relies on the cortical bone of the pedicle and both adjacent endplates to form the anchorage; therefore, it provides additional benefit in comparison with the traditional pedicle screw. Nottmeier and Pirris described transdiscal screw placement for patients with osteoporosis to improve the screw purchase in the thoracic spine by using spinal navigation..$^{9}$ For the last several years our group has been focused on transdiscal fusion constructs. ${ }^{2}$ The most challenging part of the transdiscal stabilization is finding the optimal trajectory. We have previously shown the feasibility of transdiscal trajectory in the lumbar spine even in cases without spondylolisthesis. In this paper we also report the feasibility of transdis- 
cal trajectory for the thoracic spine. The above-mentioned physiological kyphotic shape of the thoracic spine allows one to access the vertebral body through the transdiscal trajectory. Our opinion is that transdiscal stabilization in the thoracic spine can be performed safely with biplanar fluoroscopy.

\section{Conclusions}

With this first description of the technique, we could show that the transpedicular transdiscal trajectory can be performed relatively easily for PMMA cement augmentation in cases in which the classical transpedicular trajectory is not feasible. More data need to be reported to assess safety and efficacy as well as the most appropriate indication of this approach in comparison with previous standard treatment options.

\section{References}

1. Abdu WA, Wilber RG, Emery SE: Pedicular transvertebral screw fixation of the lumbosacral spine in spondylolisthesis. A new technique for stabilization. Spine (Phila Pa 1976) 19:710-715, 1994

2. Aghayev K, Gonzalez-Blohm SA, Doulgeris JJ, Lee WE III, Waddell JK, Vrionis FD: Feasibility and biomechanical performance of a novel transdiscal screw system for one level in non-spondylolisthetic lumbar fusion: an in vitro investigation. Spine J 14:705-713, 2014

3. Ge Z, Ma R, Chen Z, Zhang H, Ding H, Liang S, et al: Uniextrapedicular kyphoplasty for the treatment of thoracic osteoporotic vertebral fractures. Orthopedics 36:e1020-e1024, 2013

4. Mao H, Geng D, Zhu X, Ji S, Zhu M, Gao M, et al: Intervertebral disc degeneration induced by intradiscal poly(methyl methacrylate) leakage after spine augmentation in an in vivo rabbit model. Acta Biomater 10:3059-3067, 2014

5. Masuda K, Aota Y, Muehleman C, Imai Y, Okuma M, Thonar EJ, et al: A novel rabbit model of mild, reproducible disc degeneration by an anulus needle puncture: correlation between the degree of disc injury and radiological and histological appearances of disc degeneration. Spine (Phila Pa 1976) 30:5-14, 2005

6. Mehdizade A, Payer M, Somon T, Willhelm K, Kelekis A,
Wetzel S, et al: Percutaneous vertebroplasty through a transdiscal access route after lumbar transpedicular instrumentation. Spine J 4:475-479, 2004

7. Minamide A, Akamaru T, Yoon ST, Tamaki T, Rhee JM, Hutton WC: Transdiscal L5-S1 screws for the fixation of isthmic spondylolisthesis: a biomechanical evaluation. J Spinal Disord Tech 16:144-149, 2003

8. Nassr A, Lee JY, Bashir RS, Rihn JA, Eck JC, Kang JD, et al: Does incorrect level needle localization during anterior cervical discectomy and fusion lead to accelerated disc degeneration? Spine (Phila Pa 1976) 34:189-192, 2009

9. Nottmeier EW, Pirris SM: Placement of thoracic transvertebral pedicle screws using 3D image guidance. J Neurosurg Spine 18:479-483, 2013

10. Ringer AJ, Bhamidipaty SV: Percutaneous access to the vertebral bodies: a video and fluoroscopic overview of access techniques for trans-, extra-, and infrapedicular approaches. World Neurosurg 80:428-435, 2013

11. Ryu KS, Park CK, Kim MK, Kim DH: Single balloon kyphoplasty using far-lateral extrapedicular approach: technical note and preliminary results. J Spinal Disord Tech 20:392398, 2007

12. Sandu N, Pöpperl G, Toubert ME, Spiriev T, Arasho B, Orabi $\mathrm{M}$, et al: Current molecular imaging of spinal tumors in clinical practice. Mol Med 17:308-316, 2011

\section{Disclosures}

The authors report no conflict of interest concerning the materials or methods used in this study or the findings specified in this paper.

\section{Author Contributions}

Conception and design: all authors. Acquisition of data: all authors. Analysis and interpretation of data: all authors. Drafting the article: all authors. Critically revising the article: all authors. Reviewed submitted version of manuscript: Filis, Aghayev, Schaller, Luksza. Study supervision: Vrionis.

\section{Correspondence}

Frank Vrionis, Department of Neuro-Oncology, H. Lee Moffitt Cancer Center and Research Institute, Morsani College of Medicine, University of South Florida, 12902 Magnolia Dr., Tampa, FL 33612. email: frank.vrionis@moffitt.org. 